\title{
Exact results with the J-integral applied to free- boundary flows
}

\section{Citation}

Amar, Ben Martine, and James R. Rice. 2002. Exact results with the J-integral applied to freeboundary flows. Journal of Fluid Mechanics 461: 321-341.

\section{Published Version}

http://dx.doi.org/10.1017/S0022112002008418

\section{Permanent link}

http://nrs.harvard.edu/urn-3:HUL.InstRepos:2640594

\section{Terms of Use}

This article was downloaded from Harvard University's DASH repository, and is made available under the terms and conditions applicable to Other Posted Material, as set forth at http:// nrs.harvard.edu/urn-3:HUL.InstRepos:dash.current.terms-of-use\#LAA

\section{Share Your Story}

The Harvard community has made this article openly available.

Please share how this access benefits you. Submit a story.

Accessibility 


\title{
Exact results with the $J$-integral applied to free-boundary flows
}

\author{
By MARTINE BEN AMAR ${ }^{1}$ AND JAMES R. RICE \\ ${ }^{1}$ Department of Mechanical Engineering, Massachusetts Institute of Technology, \\ Cambridge, MA 02139, USA and Laboratoire de Physique Statistique, \\ 24 rue Lhomond, 75231 Paris Cedex 05, France \\ ${ }^{2}$ Division of Engineering and Applied Sciences and Department of Earth and Planetary Sciences, \\ Harvard University, 224 Pierce Hall, Cambridge, MA 02138, USA
}

(Received 12 January 2001 and in revised form 14 September 2001)

We apply the $J$-integral to free-boundary flows in a channel geometry such as viscous fingering or blob injection in Hele-Shaw cells, void propagation in electromigration, and injection of air bubbles into inviscid liquids. The theory of that and related conservation integrals, developed in elasticity, is outlined in a way that is applicable to fluid mechanics problems. Depending on the boundary conditions, for infinite bubbles in Laplacian fields we are able to use the $J$-integral to predict finger width if such solutions exist or to predict that there are no solutions. For finite sized bubbles, bounds can sometimes be derived. In the case of Hele-Shaw flows, in which solutions appear as a continuum, finger width cannot be constrained, but we do obtain a new derivation and generalization of Richardson moment conservation. Applications to vortex motion are also outlined briefly.

\section{Introduction}

We present here the technique of the path-independent $J$-integral (Rice $1968 a, b$ ) for interfacial fluid instabilities. The $J$-integral is commonly used in elasticity for cracks and notches in static and steady-state dynamic cases. It is useful both for its path independence, which we exploit here, and its property of giving the configurational force on an elastic singularity (Eshelby 1951, 1956; Sanders 1960; Cherepanov 1967; Rice 1968b). It allows an easy determination of the asymptotic stress and strain in the close vicinity of a crack, for example. It is not restricted to the linear elasticity case where the field in the sample is biharmonic (Eshelby 1970; Knowles \& Sternberg 1972), and can also take into account plasticity for non-growing cracks, within the approximation of 'deformation theory' (Hutchinson 1968; Rice \& Rosengren 1968). In this paper we present some applications of this technique when the field in the flow is Laplacian. We treat successively Darcy's law in the Hele-Shaw context, electromigration, and Euler flow for inviscid fluids, in the strip geometry. We consider infinite or finite bubbles in two or three dimensions but with axisymmetry. Since the boundary conditions which constrains the dynamics, and so the existence of the solution, concern derivatives of the field $\phi$ in the normal and the tangential directions, locally defined on the interface and on the cell boundaries, the $J$-integral involving $\phi$ seems to be especially convenient. The application is very simple and for an infinite finger-shaped solution, we are in some cases able to predict the asymptotic relative width of the finger compared to the cell size when it is unique 
or the possibility of a selection process by surface tension. This information does not require the complete solution of the free-boundary problem, which is very difficult and sometimes impossible, for example for electromigration and for inviscid flows with surface tension. It requires only the boundary conditions. For finite bubbles, only bounds can be derived. We also show briefly the use of path-independent integrals for the problems of injection of a fluid blob into a Hele-Shaw cell and the motion of vortex lines in two-dimensional flows of inviscid fluids.

\section{Presentation of the $J$-integral}

We consider the case of a semi-infinite finger growing steadily along the $x$-axis in an infinite strip of width $2 a$ in the $y$-direction. For the moment, we restrict ourselves to solutions that are symmetric in $y$; the case of non-symmetric fingers travelling closer to one side than the other will be examined later. The process geometry is assumed to be bidimensional. The extension to a three-dimensional geometry with axisymmetry is straightforward. We consider first that the growth is limited by a Laplacian field valid everywhere in the strip outside the finger. The normal gradient of this field vanishes along the finger and on both sides of the channel. This condition is less restrictive than it may appear: for hydrodynamic flows, it simply means that we choose the finger frame as the frame of coordinates instead of the laboratory frame. We ask the following question: What will be the relative width $\lambda$ of the infinite finger, if it exists, assuming that the Laplacian field diverges at $+\infty$ as $\phi \simeq x$. When the field diverges linearly, it is always possible to rescale to this case by a convenient choice of the units. We choose $2 a$ as the unit length so the $y$-coordinate varies between $+\frac{1}{2}$ (which corresponds to the upper-side $C_{1}$ ) and $-\frac{1}{2}$ (the lower side $C_{2}$, see figure 1). On the finger $\Gamma$, both the Neumann and Dirichlet conditions are imposed. Because of these two conditions applied simultaneously, we are faced with a non-trivial free-boundary problem where the shape of the interface cannot be prescribed arbitrarily. Thus we must solve

$$
\Delta \phi=0
$$

with $\boldsymbol{n} \cdot \nabla \phi=0$ on $\Gamma$, on $C_{1}$, and on $C_{2}$. The Dirichlet condition on $\Gamma$ depends on the process. Generically, it concerns either the value of $\phi$ or its derivative $\phi_{s}$ with respect to the arclength $s$, along the finger (here and subsequently, $\phi$ with a subscript denotes its partial derivative in the direction given by that subscript; that convention does not apply to other variables). Since the field is Laplacian, and since $\int_{C} \phi_{n} \mathrm{~d} s=0$ for any closed contour $C$, a conservation equation between far fields is obtained very easily. Calling $\boldsymbol{E}$ the gradient of $\phi$, one obtains

$$
E_{-\infty}(1-\lambda)=E_{+\infty}=1,
$$

with $E_{ \pm \infty}=\phi_{x}$ at $x= \pm \infty$. Both $E_{-\infty}$ and $E_{+\infty}$ are directed along the $x$-axis and perpendicular to the contours $C_{3}, C_{4}$ and $C_{5}$ (see figure 1). This relation is simply the conservation of the flux in hydrodynamics. More formally, it can be derived in the following way: $\Delta \phi=0$ means that

$$
\operatorname{Re}\left[\frac{\partial^{2} \Phi(z)}{\partial z^{*} \partial z}\right]=0,
$$

where $\Phi$ is the complex potential $\Phi=\phi+\mathrm{i} \chi, z$ the complex spatial coordinate $z=x+\mathrm{i} y$, and $z^{*}$ the complex conjugate.

The $J$-integral, when applied to Laplacian fields, can be obtained by a similar route. Let us consider the analytic function $(\mathrm{d} \Phi(z) / \mathrm{d} z)^{2}=\Phi^{\prime}(z)^{2}$ where a prime means the 


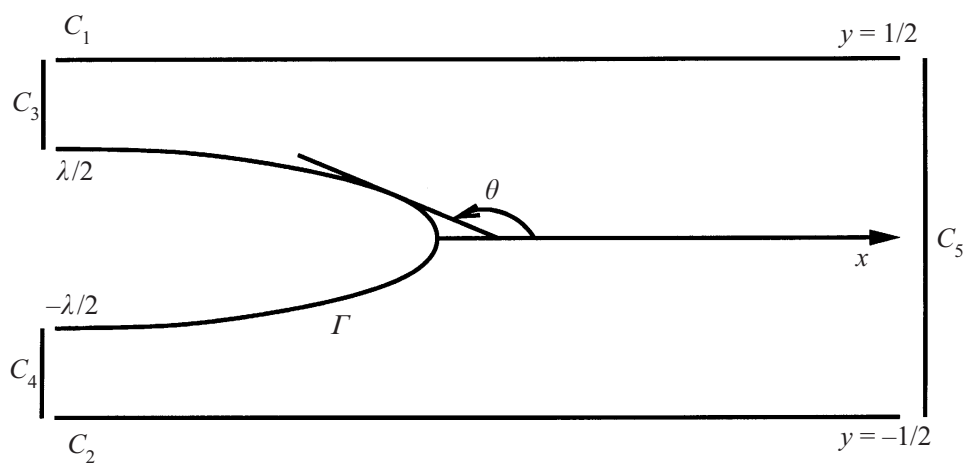

FIGURE 1. The infinite finger propagating in an infinite channel. This geometry is common to the viscous fingering case, the electromigration case and a bubble propagating into an isodensity and inviscid fluid.

derivative with respect to the natural variable $z$, and use the relation

$$
\frac{\partial \Phi^{\prime}(z)^{2}}{\partial z^{*}}=0 .
$$

Taking the real part of (2.4) one obtains the relation

$$
\frac{1}{2} \frac{\partial}{\partial x}\left[(\nabla \phi)^{2}\right]-\frac{\partial}{\partial x}\left(\phi_{x}^{2}\right)-\frac{\partial}{\partial y}\left(\phi_{x} \phi_{y}\right)=0
$$

which, when integrated over a bidimensional domain containing no singularities gives, with help of the divergence theorem, the path-invariant (and vanishing) $J$-integral as in (2.7) below. One may also see, in two or three dimensions, that in a domain where $\Delta \phi=0$

$$
\frac{\partial}{\partial x_{\alpha}}\left[\frac{1}{2}(\nabla \phi)^{2} \delta_{\alpha \beta}-\phi_{\alpha} \phi_{\beta}\right]=0
$$

(which follows by rearrangement of $(\Delta \phi)(\nabla \phi)=0$ ) giving a respective line or surface integral version associated with each coordinate direction. The $J$-integral of fracture mechanics also has a complex variable form for plane elasticity (Budiansky \& Rice 1973). For other situations like three-dimensional-Laplacian growth with axisymmetry where we cannot use the technique of analytic function theory, (2.6) suggests obvious transformations.

After integration of (2.5) over the whole domain outside the finger, we can transform the surface integral $\int \mathrm{d} x \mathrm{~d} y$ into a contour integral. For any closed contour $C$, one obtains

$$
J_{C}=\frac{1}{2} \int_{C}\left(\phi_{s}^{2}+\phi_{n}^{2}\right) \mathrm{d} y-\int_{C}\left(\phi_{x} \phi_{n}\right) \mathrm{d} s=0 .
$$

There exists another component of $J$ in the two-dimensional case when we replace $\mathrm{d} y$ with $-\mathrm{d} x$ and $\phi_{x}$ with $\phi_{y}$; that is $J$ forms a vector of two integrals in two dimensions, with there being a third component in the three-dimensional case.

Alternatively, let us consider

$$
\int\left(\phi_{x}^{2}+\phi_{y}^{2}\right) \mathrm{d} x \mathrm{~d} y
$$

or its three-dimensional generalization, the stationarity of which yields $\Delta \phi=0$. Applying the Noether theorem (Noether 1918, translated in Tavel 1971) to an invariant 
translational transformation in the $x$ (or $x_{\alpha}$ ) direction (see Gunther 1962; Knowles \& Sternberg 1972 for examples in elasticity), one derives (2.7) and the integral(s) associated with the other coordinate direction(s). This formulation has the advantage of applying, in any number of dimensions, to any field resulting from a variational principle with a translationally invariant Lagrangian density. The Lagrangian density need not be a function of quadratic order in the field variables and their derivatives, i.e. the resulting field equations need not be linear (the original applications in crack theory were principally to nonlinear elasticity). Also, this route via the Noether theorem, while not used in the discovery of the $J$-integral, has led to the discovery of a further path-independent integral $L$ when there is invariance under infinitesimal rotation about a given axis and to yet another integral $M$ when there is invariance relative to a self-similar change of scale (see Gunther 1962; Knowles \& Sternberg 1972; Budiansky \& Rice 1973; Rice 1985). For example, in the two-dimensional case of Laplacian fields, and a closed contour $C$ that surrounds no singularities, these additional integrals are

$$
M_{C}=\frac{1}{2} \int_{C}\left(\phi_{s}^{2}+\phi_{n}^{2}\right)(x \mathrm{~d} y-y \mathrm{~d} x)-\int_{C} \phi_{n}\left(x \phi_{x}+y \phi_{y}\right) \mathrm{d} s=0
$$

and

$$
L_{C}=-\frac{1}{4} \int_{C}\left(\phi_{s}^{2}+\phi_{n}^{2}\right) \mathrm{d}\left(x^{2}+y^{2}\right)-\int_{C} \phi_{n}\left(x \phi_{y}-y \phi_{x}\right) \mathrm{d} s=0 .
$$

In three dimensions, there is one (scalar) $M$-integral, whereas $L$ and $J$ are vectors of three integrals. The version of the $M$ integral corresponding to two-dimensional elasticity theory has been used to extract the strength of crack tip singularities in various cases (Freund 1978; Ouchterlony 1978, 1980; Kubo 1982) and to solve for configurational forces on dislocations (Rice 1985); we will apply the $L$ - and $M$-integrals here to two-dimensional vortex motion in inviscid fluids.

As a further example of such conservation integrals of use for fluid mechanics problems, consider Stokesian flows of possibly nonlinear fluids, with the property that the deviatoric components, $\tau_{i j}$, of the stress tensor $\sigma_{i j}=\tau_{i j}-p \delta_{i j}$ are given by $\tau_{i j}=\partial \Omega / \partial\left(\partial V_{i} / \partial x_{j}\right)$, where the flow velocities are $V_{i}$, and $\Omega$ is some function of the second and third invariants of $\partial V_{i} / \partial x_{j}+\partial V_{j} / \partial x_{i}$ (for which the first invariant vanishes by incompressibility). That includes the linear viscous case when $\Omega$ is chosen proportional to the second invariant. The existence of $\Omega$ ensures that a variational principle underlies the creeping flow problem. For all such fluids, when subjected to uniform body force fields like gravity, there exist in three dimensions vectors of three $J$-integrals and of three $L$-integrals, and if $\Omega$ is a homogeneous function of any fixed degree in $\partial V_{i} / \partial x_{j}$ ('power-law fluid') there also exists a scalar $M$-integral. These can be found from the elastic $J, L$ and $M$ (Budiansky \& Rice 1973; Knowles \& Sternberg 1972) by using the well-known analogy between the equations of elasticity with infinitesimal strains but possibly nonlinear stress-strain relations and the nonlinear viscous fluid case. For example, in the two-dimensional case, a particular component of $J$ is, for a closed contour surrounding no singularities,

$$
J_{C}=\int_{C}\left[\left(\Omega-\rho g_{\alpha} V_{\alpha}\right) \mathrm{d} y-\left(n_{\alpha} \sigma_{\alpha \beta} \partial V_{\beta} / \partial x\right) \mathrm{d} s\right]=0
$$

where there is summation over $x, y$ on the repeated indices $\alpha$ and $\beta$, and where $\rho$ is mass density, the $g_{\alpha}$ are components of gravitational acceleration, and $n_{\alpha}$ components of the unit outer normal to $C$. We do not apply that result in the present paper. 
In all cases when there are singularities within the domain, $J, L$ and $M$ take generally non-zero values which are the same for all contours surrounding the singularity point or domain.

In the case of linear field theories, additional conserved integrals can be generated based on a sum of two solutions to the governing partial differential equations, Chen \& Shield (1977). For example, in the case of Laplacian fields, if we write $\phi^{(1+2)}=\phi^{(1)}+\phi^{(2)}$, where both $\phi^{(1)}$ and $\phi^{(2)}$ are Laplacian, then by subtraction we can identify

$$
J_{C}^{(1+2)}-J_{C}^{(1)}-J_{C}^{(2)}=\int_{C}\left[\left(\phi_{n}^{(1)} \phi_{n}^{(2)}+\phi_{s}^{(1)} \phi_{s}^{(2)}\right) \mathrm{d} y-\left(\phi_{n}^{(1)} \phi_{x}^{(2)}+\phi_{n}^{(2)} \phi_{x}^{(1)}\right) \mathrm{d} s\right],
$$

which is conserved for all paths $C$ surrounding any domain within which either $\phi^{(1)}$ or $\phi^{(2)}$ has singularities, and is zero in absence of singularities. Later, we show an example of this in deriving the Richardson (1972) moments in Hele-Shaw flows with source points.

In our applications of (2.7) to fingering problems, most of the terms may vanish because of $\phi_{n}$. For instance, (2.7), when evaluated along $C_{1}$ and $C_{2}$ gives zero so finally only $\Gamma, C_{3}, C_{4}$ and $C_{5}$ contribute to the surface integral, to give

$$
\int_{-\lambda / 2}^{\lambda / 2} \phi_{s}^{2} \mathrm{~d} y=\frac{\lambda}{(1-\lambda)}
$$

Here, the integral follows the profile of the finger; $\phi_{s}$ is evaluated on the finger at height $y$. We have taken into account (2.2) for the calculation of (2.13) on $C_{3}$ and $C_{4}$. This relation may be responsible for a strong selection process, as shown later on. Although its derivation is rather simple and the geometry of the finger rather common in pattern formation, it seems to have not been recognized in the literature on interfacial fluid flow, unlike that on fracture. We now illustrate its application to various instabilities. Since it represents a conservation law, the $J$-integral can always be applied but it can provide useful information only when $\phi_{s}^{2} \mathrm{~d} y=\mathrm{d} F$ for some function $F$ defined along the finger and known at $x=-\infty$. For example, $\phi_{s}^{2}=f(y)+g(\theta) \mathrm{d} \theta / \mathrm{d} s$ would be suitable to give $\lambda$.

\section{Application to flows in Hele-Shaw cells}

\subsection{Saffman-Taylor fingering}

Our first example is one for which it is indeed the case that we obtain a true representation from the $J$-integral but no useful information in the sense of allowing us to infer some important property of the solution without solving for the full field. This emphasizes that the integral will not be helpful in all fingering problems.

The Saffman-Taylor instability occurs when a viscous fluid (oil) is pushed by a less viscous one (air) in a Hele-Shaw cell. After a short time, a long finger appears in an infinite cell with strip geometry. Due to the lubrication approximation (the gap $b$ between the two plates of the Hele-Shaw cell being small compared to other characteristic lengths such as the finger or the channel width) the viscous flow satisfies Darcy's equation which, with incompressibility, gives rise to a Laplacian velocity potential. If one neglects the surface tension, a continous family of exact analytical solutions is known (Saffman \& Taylor 1958), with all $\lambda$ between 0 and 1 possible. For Hele-Shaw flows, the continuity of the normal velocity and Laplace's law of capillarity are the two boundary conditions to apply on the interface. As a 
consequence, in the finger frame, the velocity potential is given by $\phi-U x$, where $\phi$ is the velocity potential in the laboratory frame which is proportional to the pressure according to Darcy's law and $U$ is the finger velocity. Now, we apply the $J$-integral to this case, knowing that, in dimensionless units,

$$
\phi_{s}=-\frac{1}{1-\lambda}\left[\frac{\mathrm{d} x}{\mathrm{~d} s}-\gamma \frac{\mathrm{d}^{2} \theta}{\mathrm{d} s^{2}}\right]=\frac{1}{1-\lambda}\left[\cos (\theta)+\gamma \frac{\mathrm{d}^{2} \theta}{\mathrm{d} s^{2}}\right] ;
$$

$\theta$ is the angle made by the tangent to the interface with the $x$-axis. It varies from $\frac{1}{2} \pi$ to $\pi$ as $s$ varies from 0 at the finger's nose to $\infty$ in the tail; $\gamma$, the dimensionless capillary number, is given by the ratio $b^{2} \gamma^{\prime} / 12 \mu U a^{2}$, with $\gamma^{\prime}$ the surface tension and $\mu$ the viscosity, all these quantities having physical dimensions. Using (2.13), one obtains a relation which is of little interest when one ignores the exact analytical profile since the left-hand side of (2.13) cannot be computed explicitly, independently of the form of the profile. When the profile is known, one can check that (2.13) is valid, as we have verified from the solution family of Saffman \& Taylor (1958). So in this context, the $J$-integral provides no useful information. We understand that, in the absence of surface tension, the interface shape can adapt to any $\lambda$ and the $J$-integral is not a new constraint. This is consistent with the existence of a continuum of solutions. As it is now well established, addition of surface tension (Shraiman 1986; Hong \& Langer 1986, 1987; Combescot et al. 1986; see Tanveer 2000 for a review) is necessary to select $\lambda$, in agreement with the experiment (Saffman \& Taylor 1958), which clearly shows a unique finger once the physical parameters are fixed. The surface tension gives rise to a subtle selection mechanism which has been the subject of many theoretical and numerical works (Tanveer 2000). They show that $\lambda$ is an increasing function of $\gamma$ from a lower threshold of $1 / 2$.

\subsection{Richardson moments}

An infinite set of conserved integrals has been discovered by Richardson (1972) for Hele-Shaw flows induced by injection of fluid at a point, and one might wonder if there is a relation to the class of integrals discussed here. Richardson's concern is with simply connected blobs of fluid occupying a time-dependent domain with zero pressure at their boundary. He shows that integrals $\iint z^{n} \mathrm{~d} x \mathrm{~d} y$, with $z=0$ at the injection point, are conserved for $n=1,2,3 \ldots$ and, as is evident from mass conservation, grow in proportion to the injection rate when $n=0$. These are integrals over a two-dimensional domain whereas the conserved integrals that we discuss here are along a one-dimensional path within a two-dimensional domain. For further discussion of this and related conservation integrals, see Richardson $(1972,1992)$, Tanveer \& Vasconcelos (1995), Cummings, King \& Howison (1997), Crowdy \& Tanveer (1998) and Crowdy (1999).

Our two $J$-integrals, and $L$ - and $M$-integrals, are independent of the path (but not necessarily zero) for any contour $C$ surrounding the injection point in Richardson's problem. We may apply $(2.12)$ by letting $\phi^{(1)}=\phi^{(1)}(x, y, t)$ be the solution to Richardson's injection problem, for which the corresponding complex potential $\Phi(z)$ has the form (Richardson 1972)

$$
\Phi^{(1)}(z, t)=(Q / 2 \pi) \log (z)+\Phi_{\text {reg }}^{(1)}(z, t),
$$

where $\Phi_{\text {reg }}$ is regular throughout the domain $D(t)$ occupied by the fluid, and where $\phi^{(1)}=0$ (zero pressure) on the unknown outer bounding contour $C_{o}(t)$ of the domain. We choose $\phi^{(2)}=\phi^{(2)}(x, y)$ as a function which is regular everywhere within the domain, and which satisfies no special boundary conditions, but does satisfy the 
condition $\phi_{x}^{(2)}=0$ at the injection point $z=0$. Since $\phi^{(2)}$ is regular, $J^{(2)}=0$, and it is easy to show (by shrinking the contour $C$ onto the injection point) that $J^{(1+2)}=J^{(1)}$. Thus, when we recall that $\phi_{n}^{(1)}=V_{n}$, the normal velocity of the fluid boundary $C_{o}(t)$, and that $\phi_{s}^{(1)}=0$ and hence $\phi_{x}^{(1)}=\phi_{n}^{(1)} \mathrm{d} y / \mathrm{d} s$ along $C_{o}(t)$, we obtain from (2.12)

$$
0=-\int_{C_{o}(t)} \phi_{x}^{(2)}(x, y) V_{n}(s, t) \mathrm{d} s .
$$

But we can write $\phi_{x}^{(2)}=\operatorname{Re}\left[\Phi^{(2)}(z)\right]$ and recognize that $V_{n} \mathrm{~d} s$ is just the local rate at which the area of the domain $D(t)$, occupied by the fluid, is swept out. Hence,

$$
\int_{D(t)} \operatorname{Re}\left[\Phi^{\prime(2)}(z)\right] \mathrm{d} x \mathrm{~d} y=\text { constant }
$$

for every function $\Phi^{\prime(2)}(z)$ which is analytic in the flow domain $D(t)$ over the time interval considered and which has a real part that vanishes at $z=0$. Choosing $\Phi^{\prime(2)}(z)=z^{n}$, or i $z^{n}$, with $n>0$, this provides a new derivation of Richardson's (1972) moment conservation.

We can also derive a less simple conservation result when a portion $C_{b}(t)$ of the outer contour $C_{o}(t)$ coincides with a fixed, impenetrable border of $D(t)$, so that the boundary condition changes from $\phi_{s}^{(1)}=0$ on $C_{o}(t)-C_{b}(t)$ to $\phi_{n}^{(1)}=0$ on $C_{b}(t)$. Then $\left[\phi_{x}^{(1)}, \phi_{y}^{(1)}\right]=\phi_{s}^{(1)}[\mathrm{d} x / \mathrm{d} s, \mathrm{~d} y / \mathrm{d} s]$ on $C_{b}(t)$ and (2.12) shows, after a little manipulation, that

$$
0=-\int_{C_{o}(t)-C_{b}(t)} \phi_{x}^{(2)} \phi_{n}^{(1)} \mathrm{d} s+\int_{C_{b}(t)} \phi_{y}^{(2)} \phi_{s}^{(1)} \mathrm{d} s
$$

whenever $\phi^{(2)}$ is regular in the domain $D(t)$ and $\phi_{x}^{(2)}=0$ at $z=0$. The first integral is the same as in (3.3) and this result will therefore lead again to the conservation result as stated in (3.4) if we ensure, additionally, that $\phi^{(2)}$ is yet further constrained so that $\phi_{y}^{(2)}=0$ along $C_{b}(t)$ (so that the second integral vanishes). Thus for partially blocked injection flows we obtain (3.4) for every function $\Phi^{(2)}(z)$ which is analytic in the flow domain, satisfies $\operatorname{Re}\left[\Phi^{(2)}(0)\right]=0$ and additionally satisfies $\operatorname{Im}\left[\Phi^{\prime(2)}(z)\right]=0$ on the portion of the blocking boundary which is touched by the flow domain over the time interval considered.

\subsection{Note on liquid injection into a three-dimensional porous medium}

We briefly outline the extension of (3.4) to the analogous Darcy flow case of injection, at a point and at a uniform rate, of liquid into an otherwise air-filled porous medium, to grow a liquid blob in three dimensions. This uses the three-dimensional version of the $J$-integral, for a pair of fields as in (2.12). We adopt the standard model that pore pressure is constant at the boundary of the blob, and we neglect gravity. The latter is acceptable when the injection rate is high, and/or the size of blob is small, and/or the hydraulic conductivity of the pore fluid is small. The steps of the derivation follow that of (3.4) above, extended to three dimensions in a straightforward way. Thus, one proves that

$$
\int_{D(t)} u(x, y, z) \mathrm{d} x \mathrm{~d} y \mathrm{~d} z=\text { constant }
$$

for every harmonic function $u$ which is regular in the three-dimensional region $D(t)$ occupied by the blob up to the time of interest and which vanishes at the injection point. 


\section{The $J$-integral applied to electromigration}

We call the drift of voids in a metal circuit due to the imposed electrical current electromigration. When an electrical current passes through a thin metal film, collisions between the conduction electrons and the metal ions lead to drift of the ions, in the opposite direction to the electrical field. This effect combined with the natural displacement of a positive charge in the direction of the electrical field gives an ionic current which is inhomogeneous near a void. As a consequence, due to mass conservation, the void moves in the direction opposite to the ions. So voids can deform and travel all along the metal lines. Due to the continuing miniaturization of integrated circuits, the metal lines or interconnects are subject to increasingly high current densities. Under these conditions, electromigration can be responsible of the failure of the circuit. This topic has been the subject of many publications (Arzt et al. 1994; Suo, Wang \& Yang 1994; Schimschak \& Krug 1997, 1998; Mahadevan \& Bradley 1999) but only recently has the connection to the viscous fingering instability been stressed (Ben Amar 1999; Cummings, Richardson \& Ben Amar 2001; Mahadevan \& Bradley 1999). This seems to be the perfect example to illustrate the selection by the $J$-integral on an interfacial free-boundary problem.

\subsection{The two-dimensional infinite void in the strip geometry}

We focus on the propagation of long voids (finger-shaped) in conducting thin film having the shape of a strip. Due to the imposed current and to the electron wind, the ions at the surface of the void drift. A numerical treatment (Ben Amar 1999) has shown a surprising selection for the relative width $\lambda$ in this case: $\lambda=2 / 3$. This result is obtained by integration of the interfacial equations derived after a standard classical mapping in the complex plane. The model can include a 'curvature-like effect': when a bump appears, a local diffusion of ions appears in the direction of the bump, in order to prevent cusp formation. The $2 / 3$-value is obtained in the absence of this effect. Up to now, no theoretical treatment was able to explain the existence of a unique solution. We show here that the $J$-integral offers a simple explanation.

The electric field, normalized to 1 at infinity is quasi-static so the electrical potential $\phi$ satisfies Laplace's equation. No current flows through the film edges (called $C_{1}$ and $C_{2}$ ) or through the void contour so $\phi_{n}=0$ there. The void can move in some direction when there is depletion of atoms. Depletion occurs when the current is inhomogeneous in space near the void. Since it remains tangential, for any position $\boldsymbol{R}$ on the void, due to mass conservation one has

$$
\boldsymbol{n} \cdot \frac{\mathrm{d} \boldsymbol{R}}{\mathrm{d} t}=U \frac{\mathrm{d} y}{\mathrm{~d} s}=\frac{\mathrm{d} J_{s}}{\mathrm{~d} s}=\frac{\mathrm{d}}{\mathrm{d} s}\left[M_{i}\left(Z^{*} e E_{s}-\gamma^{\prime \prime} \frac{\mathrm{d}^{2} \theta}{\mathrm{d} s^{2}}\right)\right]
$$

with $Z^{*} e$ the effective charge of the ion and $M_{i}$ the ionic mobility. The subscript $s$ on $J$ (the ionic current) and $E$ means tangential quantities. The last term in (4.1) is the stabilizing surface current which prevents cusp formation, like capillarity. Here $\gamma^{\prime \prime}$ is the surface tension (assumed isotropic) of the solid times the atomic volume. Assuming steady propagation without change of shape along the $x$-axis, one can perform an integration on (4.1) and obtain

$$
\phi_{s}-\gamma \frac{\mathrm{d}^{2} \theta}{\mathrm{d} s^{2}}=U y=\left[\frac{2}{\lambda(1-\lambda)}\right] y .
$$

Here $U$ is the dimensionless void velocity, $(2 a v) /\left(Z^{*} e M_{i} E_{0}\right)$, with $v$ the steady void 
velocity in physical units and $E_{0}$ the electrical field at plus infinity. $U$ appears as an eigenvalue fixed by the asymptotic relation (equation (2.2)).

In a first step, we neglect the capillary effect and note from (2.13) that the left-hand side can be integrated at once to give

$$
\frac{4}{(\lambda(1-\lambda))^{2}} \frac{\lambda^{3}}{12}=\frac{\lambda}{(1-\lambda)} .
$$

This has the unique solution

$$
\lambda=\frac{2}{3},
$$

which explains the observations based on numerical solutions discussed above.

It is worthwhile recalling that all the standard methods of complex analysis like the Hodograph methods with conformal mapping (Saffman \& Taylor 1958; Taylor \& Saffman 1959; McLean \& Saffman 1980) and Scharwz's method failed to find this result although they were very successful for the Saffman-Taylor problem even in unusual contexts (see for example viscous fingering in the wedge geometry, Ben Amar 1991). It turns out that the boundary condition in (4.2) seems to be responsible for the failure of such analytic function treatments since this condition breaks the 'conformal symmetry': it balances the modulus of a complex function: $E_{S}=\left[\Phi^{\prime}(z) \Phi^{\prime *}\left(z^{*}\right)\right]^{1 / 2}$ with $y$ the imaginary part of $z$. As a consequence, conformal mapping techniques like in Saffman \& Taylor (1958) give a nonlinear equation, difficult or impossible to solve. One can give another example where the traditional methods for solving explicitly free-boundary problems fail: the Bernoulli equation for a bubble growing in an inviscid and potential flow. This presents the same difficulty since it involves the square of the velocity. We will come back to this example in $\S 6$.

We can add, at least pertubatively, the capillary effect for electromigration. To leading order in $\gamma$ one obtains

$$
\lambda=\frac{2}{3}-6 A_{0} \gamma
$$

with $A_{0}$ a positive coefficient depending on the exact solution with vanishing $\gamma$. This solution is not known explicitly but it is not difficult to be convinced that

$$
A_{0}=\int_{0}^{\lambda / 2} y\left[\frac{\mathrm{d}^{2} \theta}{\mathrm{d} s^{2}}\right]_{\gamma=0} \mathrm{~d} y
$$

is a positive quantity since the integrand is positive everywhere. So, if capillarity does not destroy the solution (in other words, if a solution exists with capillarity) it acts as a regular perturbation, contrary to the Saffman-Taylor case where it implies a singular perturbation analysis. This result is in fact a direct consequence of the uniqueness of the solution without surface tension. As a consequence, the deviation from $\frac{2}{3}$ is linear in $\gamma$ while, for viscous fingering it deviates from $\frac{1}{2}$ by a $\gamma^{2 / 3}$ expansion (McLean $\&$ Saffman 1980). From our study of a limited list of examples, the technique fails to deliver a definitive value of $\lambda$ in the cases for which surface tension is a singular perturbation. Note that, for electromigration, an unphysical negative surface tension gives a finger larger than $\frac{2}{3}$, which is in agreement with the numerics. However, a positive surface tension decreases the asymptotic width of the finger and the numerics indicate that the finger solution no longer exists since it is in the range of finite-length bubbles.

\subsection{Finite-size voids}

The finger geometry is, probably, practically irrelevant, in electromigration, although a finger can be seen as a model for half of a very long bubble. So in this part, we adapt 
the previous analysis to the more realistic case of finite voids in the electromigration process (see figure 2). We will show that $2 / 3$ is in fact the upper limit for the width of a finite-size bubble. The area of a void in a metal is fixed by initial conditions or by the initial phase of nucleation. During the drift, it remains a fixed parameter in a steady or non-steady process according to (4.1). This seems to be found in experiments and it is confirmed by molecular dynamic simulations (Singubara, Utsunomiya \& Fuji 1995; Ohkubo, Hirotsu \& Nikawa 1996). When travelling steadily along the $x$-axis, in the absence of capillarity, the bubble has the symmetry $x \rightarrow-x$ and $y \rightarrow-y$. Let us now apply the $J$-integral to a contour containing half of $C_{5}$, the $x$-axis $C_{6}$ (which corresponds to $\psi=0, \psi$ being the equivalent of the stream function or the imaginary part of the complex electrical potential $w=\phi+\mathrm{i} \psi$ so $\phi_{y}=0$ ), one-fourth of the bubble from A to $\mathrm{B}$, called $\Gamma$, the vertical line equivalent to $C_{3}$ (we keep the same notation) and finally the upper-side of the strip (see figure 2). As previously, only part of the void $(\Gamma)$, the contours $C_{3}$ and $C_{4}$ contribute to the $J$-integral. Let us detail the contribution of $C_{3}$. The equivalent of (2.2) is

$$
\int_{\lambda / 2}^{1 / 2} E_{x} \mathrm{~d} y=\frac{1}{2} E_{\infty}=\frac{1}{2} E_{a v}(1-\lambda)
$$

where $E_{a v}$ is the average of the electrical field which is normal by symmetry to $C_{3}$. Let us calculate $J_{C_{3}}$ (that is the $J$-integral applied to $C_{3}$ following (2.7)):

$$
J_{C_{3}}=-\frac{1}{2} \int_{\lambda / 2}^{1 / 2}\left(E_{x}\right)^{2} \mathrm{~d} y=-\frac{1}{2} \int_{\lambda / 2}^{1 / 2}\left[\left(E_{x}-E_{a v}\right)^{2}+E_{a v}^{2}\right] \mathrm{d} y .
$$

So one obtains the following inequality for bubble voids:

$$
-J_{C_{3}} \leqslant \frac{1}{4} \frac{1}{(1-\lambda)}
$$

and as a consequence $J_{\Gamma}$ is smaller than $\lambda / 4(1-\lambda)$. But $J_{\Gamma}$ is itself given in terms of the electrical field at the top $E_{B}$ which is an unknown in the bubble case. However, we know that the extrema of analytic functions are always on a border and intuitively, one can guess from experience with electrostatics (e.g. Durand 1966) as well as with potential flow that the maximum of $E_{x}$ is at B, which is the top. So $E_{B}$ is greater than $E_{a v}$ and we can conclude that

$$
\frac{\lambda}{12(1-\lambda)^{2}} \leqslant J_{\Gamma}=\frac{1}{2}\left(\frac{2 E_{B}}{\lambda}\right)^{2} \int_{0}^{\lambda / 2} y^{2} \mathrm{~d} y \leqslant \frac{1}{4} \frac{\lambda}{1-\lambda}
$$

which proves that this $\frac{2}{3}$ limit is an upper bound for the width of voids of finite area. Since surface diffusion decreases the width, this result remains if one adds $\gamma$ (Ben Amar 1991).

\subsection{Asymmetric patterns in electromigration}

Here we consider voids travelling along the strip with constant velocity but which are closer to one side than the other (figure 3 ). In this case the $y \rightarrow-y$ symmetry is broken for the shape of the void, which allows in principle more degrees of freedom. For simplicity, we restrict attention to infinite finger voids. We know that such patterns exist in viscous flows. They have been found analytically in the case of vanishing surface tension by Taylor \& Saffman (1959). They are not seen in experiments except if one perturbs the symmetry of a cell by adding a thread, for example Couder, Gerard \& Rabaud (1986). Indeed it has been proven that isotropic surface tension 


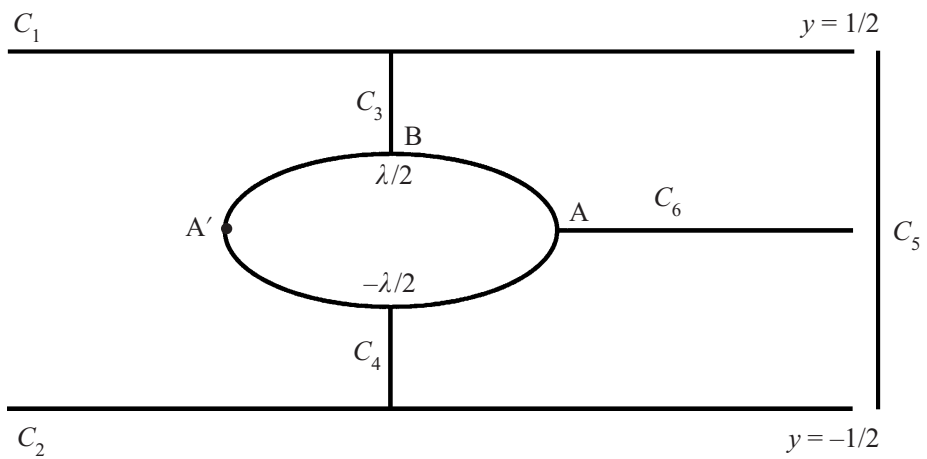

FIGURE 2. Finite-size bubble or void propagating steadily in a channel.

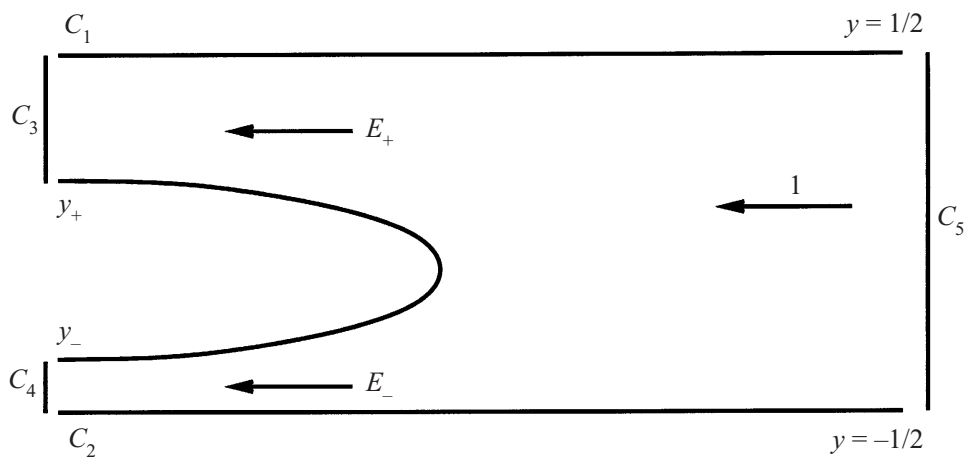

FIGURE 3. Asymmetric finger.

destroys the double continuum set of solutions found analytically. But, in the radial geometry, these solutions reappear even with isotropic surface tension. They are of great interest because of the analogy between the diffusion process and radial Hele-Shaw flow (Ben Amar \& Brener 1996). Moreover, in numerical time-dependent simulations of electromigration (M. Shelley 2000, personal communication) including surface tension, it turns out that these non-symmetric solutions have been observed.

For initial data representing a bump at one side of the cell, after a transient regime, a steady horizontal finger settles near the side where the perturbation began. Let us apply the $J$-integral to this case in order to count the available degrees of freedom. Remember that for viscous flow, this family of solutions has two degrees of freedom: $y_{0}$, some asymmetry parameter; and $\lambda$ which measures the distance between the two parallel asymptotes at $-\infty$. For non-symmetric fingers, (4.2) must be transformed since the nose of the finger is not on the central axis (see figure 3):

$$
\phi_{s}=U\left(y-y_{0}\right)=\frac{E_{+}}{y_{+}-y_{0}}\left(y-y_{0}\right)=\frac{E_{-}}{-y_{-}+y_{0}}\left(y-y_{0}\right)=\frac{E_{+}+E_{-}}{\lambda}\left(y-y_{0}\right)
$$

with $E_{+}$(resp. $E_{-}$) the electrical field at $-\infty$, close to the upper (resp. lower) side of the strip, $y_{+}$(resp. $y_{-}$) is the coordinate of the upper (resp. lower) asymptote of the finger (see figure 3). From (4.11), one can calculate $y_{+}$and $y_{-}$and keep only as unknown variables $y_{0}, \lambda, E_{+}$and $E_{-}$. The flux conservation law, (2.2), gives

$$
y_{0}\left(E_{+}-E_{-}\right)+\lambda\left(\frac{E_{+}^{2}+E_{-}^{2}}{E_{+}+E_{-}}\right)=\frac{\left(E_{+}+E_{-}\right)}{2}-1,
$$


while the $J$-integral gives, after some algebra (assuming $\gamma=0$ ),

$$
y_{0}\left(E_{+}^{2}-E_{-}^{2}\right)+\frac{4}{3} \lambda\left(\frac{E_{+}^{3}+E_{-}^{3}}{E_{+}+E_{-}}\right)=\frac{\left(E_{+}^{2}+E_{-}^{2}\right)}{2}-1 .
$$

So it may be that the asymmetry parameter $y_{0}$ and the relative width $\lambda$ are free parameters and from our analysis we may think that there is a double continuum of non-symmetric solutions, as for the viscous case. It will be interesting to see the effect of surface tension on this kind of solution. Nevertheless, the lack of symmetry gives obvious degrees of freedom, which are missing in the symmetric case, to satisfy surface tension.

\section{The $J$-integral in the three-dimensional geometry}

Instabilities occuring in two dimensions, in the Hele-Shaw cell for example, can also be observed in a tube. Nevertheless, the theoretical treatment may be rather different. As an example, for the viscous fingering in a cylindrical tube, one has to solve the three-dimensional Stokes equation instead of the Laplace equation resulting from Darcy's law. The treatment becomes tremendously difficult and often boundarylayer analyses are preferred for the prediction of pertinent parameters (Bretherton 1961). The difficulty in this particular case is two-fold: the Stokes equation in the presence of walls (here the tube) is a challenging problem even in two dimensions; second the three-dimensional geometry forbids the use of complex analysis. On the other hand the extension of the $J$-integral to the three-dimensional axisymmetric case presents no difficulty. Physically, it is difficult to imagine the electromigration process in the cylindrical geometry since it concerns thin metal films in printed circuits, but we can imagine the same process in a conducting wire. We may imagine also the following experiment: we force a finger-shaped void in a tube filled with an electrolyte solution in circumstances for which gravity plays negligible role. We can tune the physical parameters in order to suppress as much as possible the hydrodynamic effects so the charges are driven only by the electrical current. We impose an electrical current parallel to the tube. Its value at infinity is fixed to 1 . We may observe the migration of the bubble of relative radius $r=\lambda$ due to the existence of a current density. This possible experiment is somewhat similar to recent works on thermocapillary migration of bubbles in tubes (Mazouchi \& Homsy 2000, 2001; Lajeunesse \& Homsy 2002): in this case bubbles are driven by a current of matter induced by thermal change in the surface tension; the so-called Marangoni effect. According to these authors, migration of bubbles in confined geometry is rather generic and has applications in microchannels. So migration of bubbles by an electrical current may be an alternative.

As previously, we define the electrical potential, which is a function of $r$ and $z$ if the axisymmetry is preserved. The electrical current does not flow into the void so $E_{n}=\phi_{n}=0$. The mass conservation (see (4.1)) in this case is modified due to the cylindrical geometry:

$$
\boldsymbol{n} \cdot \frac{\mathrm{d} \boldsymbol{R}}{\mathrm{d} t}=U \frac{\mathrm{d} r}{\mathrm{~d} s}=\frac{1}{r} \frac{\mathrm{d}\left(r J_{s}\right)}{\mathrm{d} s}
$$

with $s$ the arclength defined by $\mathrm{d} s^{2}=\mathrm{d} r^{2}+\mathrm{d} z^{2}$, which gives a similar equation for $\phi_{s}$,

$$
\phi_{s}=\frac{1}{2} U r=\left[\frac{2}{\lambda\left(1-\lambda^{2}\right)}\right] r .
$$


Equation (2.5) also has to be modified, like (2.6) which leads to

$$
\frac{1}{2} \frac{\partial}{\partial z}\left(\nabla \phi^{2}\right)-\frac{\partial}{\partial z}\left(\phi_{z}^{2}\right)-\frac{1}{r} \frac{\partial}{\partial r}\left(r \phi_{r} \phi_{z}\right)=0 .
$$

This equality is then responsible for a conserved quantity

$$
J_{\Sigma}=\frac{1}{2} \int_{\Sigma}\left(\phi_{s}^{2}+\phi_{n}^{2}\right) 2 \pi r \mathrm{~d} r-\int_{\Sigma}\left(\phi_{z} \phi_{n}\right) \mathrm{d} S=0,
$$

where $\Sigma$ is a closed surface. This relation can be found by integration of (2.6) over the domain outside the void. On the void a simple calculation gives

$$
J_{B}=\pi \frac{1}{4} \frac{\lambda^{2}}{\left(1-\lambda^{2}\right)^{2}},
$$

which must balance the contribution given by the vertical surface which closes the fluid domain at $-\infty$ and $+\infty$ :

$$
\frac{\pi}{2} \frac{\lambda^{2}}{1-\lambda^{2}} .
$$

So finally one obtains for $\lambda^{2}$, the relative area of the bubble compared to the tube, $\lambda^{2}=1 / 2$.

Although the selected value is different, the logic of the selection and the calculation is the same as for the two-dimensional case. One may expect similar results for bubbles of finite size in cylindrical geometry with an upper bound for width given by $1 / \sqrt{2}$.

\section{The $J$-integral for inviscid flows}

Due to the form of the $J$-integral, and of the Bernoulli relation which involves the square of the velocity, and so $\phi_{s}^{2}$, inviscid fluid flows should be a good candidate for an easy determination of the relative width. We will consider two cases, one with gravity and the other without gravity.

\subsection{Finger in a horizontal channel}

We imagine the following situation: in an horizontal and infinite channel of width $h$ taken as unit length, we push an inviscid gas into an inviscid liquid. We suppose that the channel is closed at $x \rightarrow+\infty$, so that the liquid velocity (in the laboratory frame) is zero there, but that liquid can be displaced at $x=-\infty$ to accommodate the volume of the injected gas. For example, the vertical channel wall at $x=-\infty$ may be a movable piston through which we inject gas via a hole towards the top of the wall. The channel is infinite in the transverse $z$-direction, so the description of the flow is limited to the plane $(x, y)$ (see figure 4). Since the gas (considered incompressible and of negligible density) is much lighter than the fluid, it will travel in the cell along the upper wall defined by $y=0$ while the lower one is located at $y=-1$. The bottom of the gas layer is at $y=-\lambda$. In real fluids, there may be a thin film between the wall and the bubble, but we neglect it. We assume that the dynamics is steady and irrotational, the interface evolving with a velocity $V_{i}$ in the laboratory frame, which is also our unit for velocity. The over-pressure in the gas is $p_{i}$ compared to the pressure at $+\infty$ on the cell upper surface at $y=0$. Thus, in the laboratory frame the gas velocity is $V_{i}$ and the liquid velocity is 0 at $+\infty$ but is $-\lambda V_{i} /(1-\lambda)$ at $x=-\infty$, for mass conservation. We work in the bubble, or finger, frame in which the gas velocity is 0 , and the liquid velocity is $-V_{i}$ at $+\infty$ and $-V_{i} /(1-\lambda)$ at $-\infty$. The fluid velocity 
is potential and, on the interface, we must satisfy the Bernoulli relation

$$
\frac{1}{2} v^{2}+\gamma \frac{\partial \theta}{\partial s}+\frac{y}{F}=B
$$

with $F$ the Froude number, a dimensionless gravity parameter given by $V_{i}^{2} / g h, \gamma$ is the capillary parameter given by $\gamma^{\prime} / \rho_{l} V_{i}^{2} h$ with $\rho_{l}$ the liquid density; $\gamma$ is the inverse of the Weber number. The second term on the left-hand side of (6.1) represents the pressure gap between the liquid just below the interface and $p_{i}$, the pressure inside the gas, divided by the fluid density. It must be negative. $B$ is the Bernoulli constant which holds everywhere in the fluid. The mass conservation gives the velocity of the fluid at $-\infty$ as $v_{\infty}=-1 /(1-\lambda)$. Since the normal velocity in the finger frame vanishes, we can easily apply the $J$-integral and we obtain for the bubble contribution, with $v=\phi_{s}$

$$
\frac{1}{2} \int_{-\lambda}^{0} \phi_{s}^{2} \mathrm{~d} y=B \lambda+\frac{1}{2 F} \lambda^{2}-\gamma[\cos (\theta)]_{0}^{\pi}=B \lambda+\frac{1}{2 F} \lambda^{2}+2 \gamma .
$$

$\theta$, the angle between the tangent at the interface and the central axis, varies between 0 at $-\infty$ and $\pi$ in the case of a thin film between the wall and the gas. Otherwise, it is limited by the contact angle value. It is important to notice that the $J$-integral can treat the capillary term explicitly for inviscid flow. We can calculate the Bernoulli constant $B$ from the conditions at $-\infty$ as

$$
B=\frac{1}{2} \frac{1}{(1-\lambda)^{2}}-\frac{\lambda}{F}
$$

and hence write the bubble contribution of (6.2) as

$$
\frac{\lambda}{2(1-\lambda)^{2}}-\frac{\lambda^{2}}{2 F}+2 \gamma
$$

Finally taking into account the $J$-integral at both ends (on the contours $C_{3}$ and $C_{5}$ ), one obtains

$$
\begin{gathered}
-\left[\frac{\lambda}{2(1-\lambda)^{2}}-\frac{\lambda^{2}}{2 F}+2 \gamma\right]-\frac{1}{2}+\frac{1}{2} \frac{1}{1-\lambda}=0, \\
\frac{1}{(1-\lambda)^{2}}=\frac{1}{F}-4 \frac{\gamma}{\lambda^{2}} .
\end{gathered}
$$

Now recall that $F=V_{i}^{2} / g h$, but that $V_{i}$ is not an experimentally specifiable parameter. Rather, we could specify $h Q$, the volumetric rate of gas injection per unit thickness. Since, in the finger frame, the movable partition at $x=-\infty$ moves with velocity $-V_{i} /(1-\lambda)$, the finger elongates at rate $V_{i} /(1-\lambda)$ and, since it has height $\lambda h, Q=\lambda V_{i} /(1-\lambda)$. Also, in terms of $Q, \gamma$ is $\gamma^{\prime} \lambda^{2} / \rho_{l} h Q^{2}(1-\lambda)^{2}$. Thus the forgoing solution is

which means that

$$
\frac{1}{(1-\lambda)^{2}}=\frac{g h \lambda^{2}}{Q^{2}(1-\lambda)^{2}}-\frac{4 \gamma^{\prime}}{\rho_{l} h Q^{2}(1-\lambda)^{2}}
$$

$$
\lambda=\sqrt{\left(Q^{2}+4 \gamma^{\prime} / \rho_{l} h\right) / g h},
$$

which expresses $\lambda$ in terms of the injection rate. We see that when $\gamma^{\prime}=0$, the maximum rate for which the finger geometry is tenable (i.e. $\lambda \rightarrow 1$ ) is $Q_{\max }=\sqrt{g h}$. Also, when we neglect surface tension, the solution for $\lambda$ is simply $\lambda=Q / \sqrt{g h}$. 


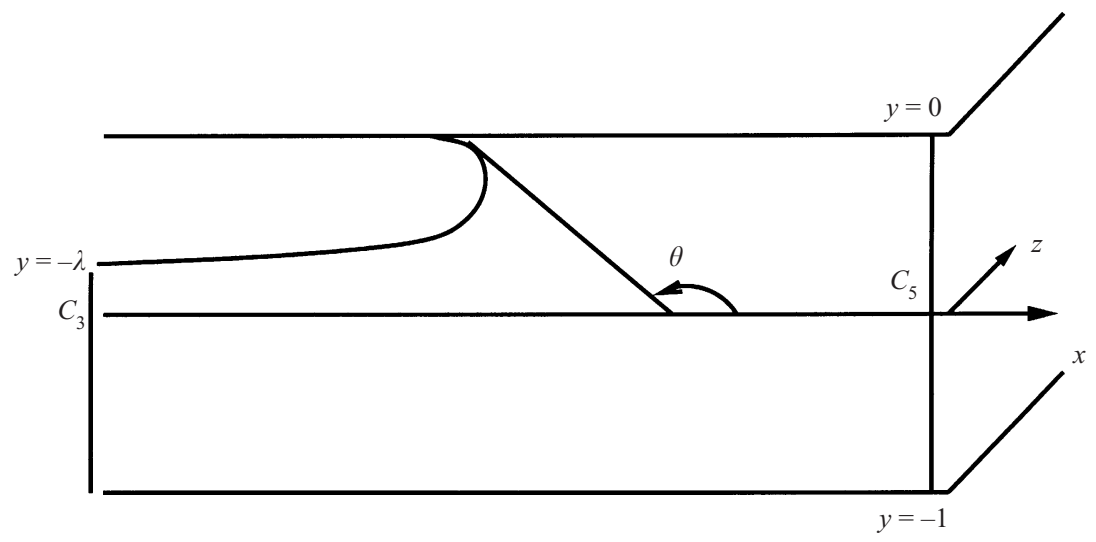

Figure 4. Weightless bubble in inviscid flow.

The two most obvious effects of capillarity are to give a non-zero minimum width $\lambda$ at zero injection rate $(Q \rightarrow 0)$

$$
\lambda_{\min }=\sqrt{4 \gamma^{\prime} / \rho_{l} g h^{2}},
$$

which would describe quasi-static enlargement of a finger-like bubble, and to limit the maximum injection rate (for a finger-like solution to occur) to the value

$$
Q_{\max }=\sqrt{g h\left(1-\lambda_{\text {min }}^{2}\right)},
$$

when $\lambda \rightarrow 1$ and the channel is completely spanned by the finger. For sufficiently small channel height $h$, namely $h<\sqrt{4 \gamma^{\prime} / \rho_{l} g}, \lambda_{\text {min }}$ cannot be greater than 1 , and so no finger-like solution can exist. Presumably, in that range, a quasi-statically enlarging gas bubble touches both the upper and lower walls of the channel. To further understand this solution, if it exists (we have not proven that it does), note again that the velocity of the fluid in the finger frame at $x=-\infty$ is $-V_{i} /(1-\lambda)=-Q / \lambda$. When $\gamma^{\prime}=0$, this is always equal to $-\sqrt{g h}$, independently of the injection rate. The local Froude number there in the finger frame is thus $F_{l o c}=\left[V_{i} /(1-\lambda)\right]^{2} / g(1-\lambda) h=1 /(1-\lambda)=1 / \sqrt{F}>1$ where $F$ is the Froude number at $x=+\infty$ and we observe that since $1-\lambda=\sqrt{F}$, finger-like solutions can exist, if at all, only for $F<1$.

We could equally have assumed that the channel end at $x=-\infty$ where injection occurs was fixed and that the end at $x=\infty$ was a movable partition, subjected to the same force as before injection. Then, if $V_{i}$ is again the interface velocity in the laboratory frame, the velocities in the finger frame are $-V_{i}$ at $x=-\infty$ and $-(1-\lambda) V_{i}$ at $x=\infty$, and the injection rate is $Q=\lambda V_{i}$. In that case, we obtain, from conditions at $-\infty$, that $B=\frac{1}{2}-\lambda / 2 F$ and the $J$-integral evaluation gives

$$
-\left[\lambda / 2-\lambda^{2} / 2 F+2 \gamma\right]-(1-\lambda)^{2} / 2+(1-\lambda) / 2=0 .
$$

Simplifying, in that case, we obtain

$$
F=\lambda^{2} /\left(\lambda^{2}+4 \gamma\right)
$$

(or just $F=1$, independently of $\lambda$, when $\gamma=0$ ). In terms of the injection rate $Q=\lambda V_{i}$ and $\gamma=\gamma^{\prime} \lambda^{2} / \rho_{l} h Q^{2}$ for this case, this again gives the same solution:

$$
\lambda=\sqrt{Q^{2}+\left(4 \gamma^{\prime} / \rho_{l} h\right) / g h} .
$$




\subsection{Momentum interpretation of the $J$ - and L-integrals}

We note that in the inviscid fluid case, with irrotational flow, invariance with respect to contour $C$ of the $J$-integrals associated with the $x$ - and $y$-directions may be interpreted as statements of conservation of momentum. For example, neglecting body forces and working in real physical units, we may write $-p / \rho$ for $\frac{1}{2}(\nabla \phi)^{2}$ and see that the $J$-integral associated with the $x$-direction is

$$
\rho J_{C}=-\int_{C} p \mathrm{~d} y-\int_{C} \rho V_{n} V_{x} \mathrm{~d} s .
$$

The equality of $J$ for any two contours $C_{1}$ and $C_{2}$, which contain between them no embedded body (like an airfoil) or other singularity, simply expresses that the pressure force in the $x$-direction on the fluid between $C_{1}$ and $C_{2}$ equals the rate of change of its momentum in the $x$-direction. If there is an embedded body (at the boundary of which $V_{n}=0$ ) then the two $J$-integrals, associated with the $x$ - and $y$-directions, give the $x$ - and $y$-components of force $/ \rho$ (per unit thickness) acting on the body. This result, when re-expressed in terms of the complex flow potential $\Phi(z)$, simply coincides with the Blasius expression for lift and drag. Similarly, the $L$-integral relates to torque of pressure forces and the rate of change of angular momentum, and coincides with the Blasius torque expression, although we have found no classical interpretation of the $M$-integral.

\subsection{Propagating fingers in an inviscid fluid in the absence of gravity}

We imagine the same experiment as previously but with two inviscid fluids with the same density (or approximatively) in order to neglect gravity. In this case, the propagation is expected to be along the axis of symmetry of the cell or of the tube. Contrary to gravity, we assume that the capillary effects are not negligible. The frame of coordinates is the same as in figure 1 . With the help of the $J$-integral we will show that we cannot find a finger-shape steady solution to this problem in two or three dimensions, a result which may be considered as surprising. The fluid velocity inside the finger $V_{i}$ is uniform and equal to the interface velocity. In the frame of the finger, the Bernoulli law is given by (6.1) with $1 / F^{*}=0$. Equation (6.2) also applies with

$$
B=\frac{1}{2} \frac{1}{(1-\lambda)^{2}} \text {. }
$$

So one easily derives the following contradictory relation:

$$
\frac{1}{2} \frac{\lambda^{2}}{(1-\lambda)^{2}}+2 \gamma=0
$$

which shows that the unique solution with vanishing width $(\lambda=0)$ is destroyed by surface tension. The same calculation in the tube geometry gives the same answer. The $J$-integral along the axisymmetric finger with surface tension included gives

$$
\pi B \lambda^{2}+2 \pi \gamma \lambda=\frac{\pi}{2} \frac{\lambda^{2}}{\left(1-\lambda^{2}\right)^{2}}+2 \pi \gamma \lambda=\frac{\pi}{2} \frac{\lambda^{2}}{1-\lambda^{2}}
$$

using (5.6). In (6.16), we have taken into account that the curvature is given by

$$
\frac{1}{r} \frac{\mathrm{d}}{\mathrm{d} r} \frac{r z^{\prime}}{\left(1+z^{\prime 2}\right)^{1 / 2}}
$$

in cylindrical coordinates, $z^{\prime}$ being the first derivative of $z(r)$ with respect to $r$. 
The conclusion is the same as for the two-dimensional case. The only solution is $\lambda=0$. Since infinite long bubbles cannot propagate steadily, let us now examine finite-size bubbles. As previously all the calculations are made in the bubble frame. The bubble keeps the symmetry $y \rightarrow-y$ but loses the symmetry $x \rightarrow-x$, so the right and the left sides of the bubble are different. This is due to the surface tension effects. So a priori we have two tips (A and $\mathrm{A}^{\prime}$ ) with negative curvature $\left(\kappa_{1}\right)$ but two tops (B and $\left.\mathrm{B}^{\prime}\right)$ with curvature $\left(\kappa_{2}\right)$ symmetric with respect to the $x$-axis but not located at $x=0$. In this case the Bernoulli relation gives

$$
\frac{1}{2} \phi_{s}^{2}+\gamma \frac{\partial \theta}{\partial s}=B=\gamma \kappa_{1},
$$

which simply shows that the curvature at the top is larger in absolute value than that at the tip. Using the theorem of an extremum of an analytical function we find the following bound for the difference in curvature between tip and top:

$$
\frac{1}{2} \frac{1}{(1-\lambda)^{2}} \leqslant \gamma\left(\kappa_{1}-\kappa_{2}\right) .
$$

It suggests that the bubble is perhaps more elongated along the $y$-axis than along the $x$-axis.

Having integrated along the contour between $\mathrm{B}$ and $\mathrm{B}^{\prime}$, one obtains for the $J$-integral

$$
J_{B}=\gamma \kappa_{1} \lambda+2 \gamma .
$$

As for the finite-size voids of electromigration, this result has an upper bound:

$$
J_{B} \leqslant \frac{1}{2} \frac{\lambda}{(1-\lambda)} .
$$

We cannot go further, but this analysis, which involves as degree of freedom the curvature so the shape of the bubble itself, suggests that solutions may exist.

\section{Some applications to vortices in two-dimensional flows}

Some applications of path-independent integrals to straight vortex lines in two-dimensional inviscid and otherwise irrotational flow fields are given here. Most of these are well-known results which can be derived (Saffman 1992) by using the integral representations of conservation of linear and angular momentum, with which the $J$ - and $L$-integrals coincide in that case. Other results, apparently new or little known, have proofs that parallel applications of the path-independent integrals to dislocation lines in two-dimensional elasticity (Eshelby 1951, 1956, 1970; Rice 1985). Hence results are stated with little development. Again $\phi$ is the velocity potential with $V=\nabla \phi . \Gamma$, which is not to be confused with its earlier use for the finger profile, denotes the circulation (anti-clockwise) around a vortex line, and is conserved in the flow. $T$ is the kinetic energy of the flow field per unit thickness perpendicular to the flow domain.

As for a dislocation line in two-dimensional elasticity, $T$ becomes infinite in the line vortex limit and is given by $\left(\rho \Gamma^{2} / 4 \pi\right) \log \left(r_{o} / r_{i}\right)$ (Saffman 1992) where $r_{o}$ scales with a characteristic size of the flow domain and $r_{i}$ is an inner cut-off radius, interpretable as the radius of a small core of uniform vorticity. However, derivatives of $T$ with respect to the position of the vortex line are nevertheless finite and well-defined so long as $\Gamma$ and $r_{i}$ are the same for all positions considered. Let $x=\xi, y=\eta$ be the momentary position of the vortex line. The line will move in some way with the fluid but for 
the present we take the point of view that we can specify arbitrarily a momentary position of the line and ask how $T$ changes when we change that position, keeping $\Gamma$ and $r_{i}$ fixed. Assuming that contour $C$ surrounds that line and no other singularities, one may show that

$$
\rho J_{x}=-\partial T / \partial \xi, \quad \rho J_{y}=-\partial T / \partial \eta .
$$

These are analogous to the result that the configurational force on an elastic dislocation line, which is defined as the rate of decrease of potential energy of a loaded elastic system with respect to the dislocation position, is given by $\left(J_{x}, J_{y}\right)$ for that case (Eshelby 1951, 1956, 1970). By shrinking $C$ onto the vortex line and evaluating the integrals from the singular field which then dominates, one shows also that, when $r_{i}$ approaches 0 ,

$$
J_{x}=\Gamma V_{y}^{r e g}, \quad J_{y}=-\Gamma V_{x}^{r e g},
$$

where $\left(V_{x}^{r e g}, V_{y}^{r e g}\right)$ is the regular part of the velocity field at the vortex position, which is also the velocity of the line in its natural motion. The same result is given by Saffman (1992) when it is recognized that $\rho\left(J_{x}, J_{y}\right)$ is his linear momentum integral, and has an interpretation in anti-plane elasticity as the configurational force on a screw dislocation line (in which case $\Gamma$ corresponds to the Burgers vector and $\left(V_{x}, V_{y}\right)$ to the anti-plane components of shear stress).

Equation (7.2) shows that the vortex velocity $\left(V_{x}^{r e g}, V_{y}^{r e g}\right)$ is orthogonal to $\left(J_{x}, J_{y}\right)$, whereas (7.1) shows that the latter vector is directed along the gradient of the kinetic energy. One therefore concludes that the natural motion of the vortex conserves kinetic energy. The same can also be derived from the Hamiltonian structure of the equations of vortex motion (e.g. Saffman 1992) in which case the time invariance of the Hamiltonian, for fixed borders of the flow domain like we consider here, gives the result. The net pressure force on those borders is given as $\rho\left(J_{x}, J_{y}\right)$ and hence is perpendicular to the momentary velocity.

Also, by direct evaluation on a contour $C$ surrounding a vortex at $(\xi, \eta)$, and shrunk onto it, one finds that the path-independent integrals $L$ and $M$ of (2.10), (2.9), taken relative to the coordinate origin at $(0,0)$, are

$$
L=\xi J_{y}-\eta J_{x}=r J_{\theta}, \quad M=\xi J_{x}+\eta J_{y}+\Gamma^{2} / 4 \pi=r J_{r}+\Gamma^{2} / 4 \pi .
$$

The last term in $M$ may be recognized as $1 / \rho$ times the pre-logarithmic factor in the kinetic energy expression, analogous to the result of Rice (1985) for a line elastic dislocation. The polar coordinate notation denotes components of $J$ along directions that are parallel, for $J_{r}$, and perpendicular, for $J_{\theta}$, to the line of length $r$ from $(0,0)$ to $(\xi, \eta)$, and in terms of these directions, $J_{r}=\Gamma V_{\theta}^{\text {reg }}$ and $J_{\theta}=-\Gamma V_{r}^{\text {reg }}$.

As an elementary application, if the flow domain is a circular annulus between concentric circles and $C$ is chosen as the border of the domain, then it is immediately obvious from (2.10) that $L=0$. Thus (7.3) requires that $J_{\theta}=0$, hence confirming the expected result that $V_{r}^{\text {reg }}=0$.

Suppose now that the flow domain is bounded by an infinite wedge with vertex at $(0,0)$, and that the vortex line is distance $r$ away at $(\xi, \eta)$. Take $C$ to start at distance $r_{1}<r$ from the vertex on one flank of the wedge, to continue along that flank to a large distance $r_{2}>r$, then to follow a circular arc of radius $r_{2}$ until reaching the other flank, then to move in along that flank to radius $r_{1}$, and finally to close as a circular arc of radius $r_{1}$. From (2.9) there is no contribution to $M$ along the flanks, and none from the circular arcs either, which can be seen by letting $r_{1}$ approach 0 (the vertex singularity is too weak to contribute to $M$ ) and $r_{2}$ approach $\infty$ (the presence of the wedge boundary makes the far velocity decay faster than $1 / r$ ). Thus $M=0$, so that 
(7.3) yields $J_{r}=-\Gamma^{2} / 4 \pi r$. Combining that with $J_{r}=\Gamma V_{\theta}^{r e g}$ then gives the unexpected result that $V_{\theta}^{r e g}=-\Gamma / 4 \pi r$ for any wedge angle and any angular position $\theta$ within the flow field. The result, analogous to one in elastic dislocation theory (Rice 1985), may be checked by a conformal mapping solution.

When the wedge angle is $\pi$, in the form of a wall along the $y$-axis which bounds the flow field, use of the $J_{y}$-integral for a contour $C$ like that just considered shows that $J_{y}=0$ and hence that $V_{x}^{\text {reg }}=0$. The $M=0$ result above then shows that $V_{y}^{r e g}=-\Gamma / 4 \pi \xi$, a result which may be checked by a simple image construction (e.g. Saffman 1992).

\section{Conclusion}

As shown in this paper, the $J$-integral can be useful for predicting information on the size of bubbles when the strip geometry is considered. This geometry is very common in laboratories and is also relevant for microelectronics and micromechanics (MEMS). Unfortunately many analytical treatments, that work in the infinite geometry, fail in the strip geometry: this is so for the case of electromigration (in spite of the analyticity of the field), of the Stokes's equation which is biharmonic, and of the diffusion equation. The $J$-integral is especially efficient for a certain class of boundary conditions, the electromigration case giving the best illustration. This restriction is in fact typical of free-boundary problems where a technique suitable for one situation completely fails in another case. As an example, conformal mapping techniques McLean \& Saffman (1980), Saffman \& Taylor (1958) and Taylor \& Saffman (1959) have been shown to be efficient only for the isobaric interface. The $J$-integral provides exact results for infinite voids and bounds for finite-size voids. There are wider possibilities for applications to fluid phenomena as shown, e.g. by our brief discussion of fluid injection into Hele-Shaw cells and vortex motion. Another application (not done here) of this technique will be to solitary waves propagating along boundaries if one is interested in bounds. This technique is not limited to the examples treated in this paper, nor to problems which are harmonic or linear. Perhaps one may think of a better choice for $\phi$ in order to derive new results.

M. B. A. acknowledges the support of the McCarthy Professorship and the hospitality of the Department of Mechanical Engineering at MIT where this work was initiated. J. R. R. acknowledges support of a visiting appointment of the University of Paris VI in the Laboratoire de Physique Statistique. We are grateful to a reviewer for mentioning a possible connection between Richardson moments and the $J$-integral.

\section{REFERENCES}

Arzt, E., Kraft, O., Nix, W. D. \& Sanchez, J. E. 1994 Electromigration failure by shape change of voids in bamboo lines. J. Appl. Phys. 76, 1563-1571.

Ben Amar, M. 1991 Viscous fingering in the wedge. Phys. Rev. A 44, 3673-3685.

Ben Amar, M. 1999 Void electromigration as a moving boundary problem. Physica D 134, 275-286.

Ben Amar, M. \& Brener, E. 1996 Laplacian and diffusional growth: A unified theoretical description for symmetrical and parity-broken patterns. Physica D 98, 128-138.

Bretherton, F. P. 1961 The motion of long bubbles in tube. J. Fluid Mech. 10, 166-188.

Budiansky, B. \& Rice, J. R. 1973 Conservation laws and energy release rates. Trans. ASME: J. Appl. Mech. 40, 201-203.

Chen, F. H. K. \& Shield, R. T. 1977 Conservation laws in elasticity of $J$-integral type. Z. Angew. Math. Phys. 28, 1-22. 
Cherepanov, G. P. 1967 Crack propagation in continuous media. Appl. Math. Mech. (translation of Prikl. Mat. Mek.) 31, 476-488.

Combescot, R., Dombre, T., Hakim, V., Pomeau, Y. \& Pumir, A. 1986 Shape selection for SaffmanTaylor fingers. Phys. Rev. Lett. 56, 2036-2039.

Couder, Y., Gerard, N. \& Rabaud, M. 1986 Narrow fingers in the Saffman-Taylor instability. Phys. Rev. A 34, 5175-5178.

Crowdy, D. G. 1999 A note on viscous sintering and quadrature identities. Eur. J. Appl. Maths 10, 623-634.

Crowdy, D. G. \& TAnveER, S. 1998 A theory of exact solutions for plane viscous blobs. J. Nonlinear Sci. 8(3), 261-279.

Cummings, L. J., King, J. R. \& Howison, S. D. 1997 Conserved quantities in Stokes flow with free surfaces. Phys. Fluids 9, 477-480.

Cummings, L. J., Richardson, G. \& Ben Amar, M. 2001 Mathematical theory of void electromigration. Eur. J. Appl. Maths 12, 97-134.

Durand, E. 1966 Electrostatique, Problèmes Généraux Conducteurs. Paris: Masson.

Eshelby, J. D. 1951 The force on an elastic singularity. Phil. Trans. R. Soc. Lond. A 244, 87-112.

Eshelby, J. D. 1956 The continuum theory of lattice defects. In Progress in Solid State Physics (ed. F. Seitz \& D. Turnbull), pp. 79-114. Academic.

Eshelby, J. D. 1970 Energy relations and energy-momentum tensor in continuum mechanics. In Inelastic Behavior of Solids (ed. M. F. Kanninen et al.), pp. 77-115. McGraw-Hill.

FREUND, L. B. 1978 Stress intensity factor calculations based on a conservation integral. Intl J. Solids Struct. 14, 241-250.

Gunther, W. 1962 Ber einige randintegrale der elastomechanik. Abh. Braunschw. Wiss. Ges. 14, 53-72.

Hong, D. C. \& LANGER, J. S. 1986 Analytic theory for the selection of Saffman-Taylor finger. Phys. Rev. Lett. 56, 2032-2035.

Hong, D. C. \& Langer, J. S. 1987 Pattern selection and tip perturbations in the Saffman-Taylor problem. Phys. Rev. A 36, 2325-2332.

Hutchinson, J. W. 1968 Singular behavior at the end of a tensile crack in a hardening material. J. Mech. Phys. Solids 16, 13-31.

Knowles, J. K. \& Sternberg, E. 1972 On a class of conservation laws in linearized and finite elastostatics. Arch. Rat. Mech. Anal. 44, 187-211.

Kubo, S. 1982 An application of M-integral to cracks in dissimilar elastic materials. Intl J. Fracture 20, R27-R30.

Lajeunesse, E. \& Homsy, G. M. 2002 Thermocapillarity migration of long bubbles in polygonal capillary tube, Part 2: Experiments. Phys. Fluids (submitted).

Mazouchi, A. \& Homsy, G. M. 2000 Bubbles in cylindrical capillary tubes. Phys. Fluids 12, 542-549.

Mazouchi, A. \& Homsy, G. M. 2001 Thermocapillarity migration of long bubbles in polygonal capillary tube, Part 1: Theory. Phys. Fluids 13, 1594-1600.

Mahadevan, M. \& Bradley, R. M. 1999 Simulations and theory of electromigration-induced slit formation in unpassivated single crystal metal lines. Phys. Rev. B 59, 11037-11046.

McLean, J. W. \& SAFFman, P. G. 1980 The effect of surface tension on the shape of fingers in a Hele-Shaw cell. J. Fluid Mech. 102, 455-469.

Noether, E. 1918 Invariante variations-probleme. Nachr. Ges. Gottingen, Math. Phys. Klasse 235.

Ohkubo, T., Hirotsu, Y. \& Nikawa, K. 1996 Molecular dynamics simulations of electromigration in nano-sized metal lines. Mater. Trans. Japan Inst. Met. 37 (3), 454-457.

OuCHTERLONY, F. 1978 Some stress intensity factors for self-similar cracks derived from pathindependent integrals. J. Elasticity 8, 259-271.

OuCHTERLONY, F. 1980 Symmetric cracking of a wedge by transverse displacement. J. Elasticity 10, 215-223.

RICE, J. R. 1968a A path-independent integral and the approximate analysis of strain concentration by notches and cracks. Trans. ASME: J. Appl. Mech. 35, 379-386.

RicE, J. R. $1968 b$ Mathematical analysis in the mechanics of fracture. In Fracture: An Advanced Treatise (ed. H. Liebowitz), pp. 191-311. Academic.

RicE, J. R. 1985 Conserved integrals and energetic forces. In Fundamentals of Deformation and 
Fracture (Eshelby Memorial Symposium) (ed. B. A. Bilby, K. J. Miller \& J. R. Willis), pp. 33-56. Cambridge University Press.

Rice, J. R. \& Rosengren, G. F. 1968 Plane strain deformation near a crack in a power law hardening material. J. Mech. Phys. Solids 16, 1-12.

RichaRdSON, S. 1972 Hele-Shaw flows with a free boundary produced by the injection of fluid into a narrow channel. J. Fluid Mech. 56, 609-618.

RichaRdSON, S. 1992 Two-dimensional slow viscous flows with time-dependent free boundaries driven by surface tension. Eur. J. Appl. Maths 3, 193-207.

Saffman, P. G. 1992 Vortex Dynamics. Cambridge University Press.

SAFFMAN, P. G. \& TAYLOR, G. I. 1958 The penetration of a fluid into a porous medium or Hele-Shaw cell containing a more viscous liquid. Proc. R. Soc. Lond. 245, 312-329.

SANDERS, J. L. 1960 On the Griffith-Irwin fracture theory. Trans. ASME: J. Appl. Mech. $27,352$.

SCHIMSCHAK, M. \& KRUG, J. 1997 Surface electromigration as a moving boundary value problem. Phys. Rev. Lett. 78, 278-281.

SCHIMSCHAK, M. \& KRUG, J. 1998 Electromigration-induced breakup of two-dimensional voids. Phys. Rev. Lett. 80, 1674-1677.

Shraiman, B. 1986 On velocity selection and the Saffman-Taylor problem. Phys. Rev. Lett. 56, 2028-2031.

Singubara, S., UtsunomiYa, I. \& Fuji, T. 1995 Molecular dynamic simulations of void electromigration under a high-density electric current stress in an aluminium interconnection. Electr. Commun. Japan 278 (12), 82-95.

SuO, Z., WANG, W. \& YANG, M. 1994 Electromigration instability: Transgranular slits in interconnects. Appl. Phys. Lett. 64, 1944-1946.

TANVEER, S. 2000 Surprises in viscous fingering. J. Fluid Mech. 409, 273-308.

TANVEer, S. \& VASCONCElos, G. 1995 Time evolving bubbles in two-dimensional Stokes flow. J. Fluid Mech. 301, 325-344.

TAVEL, M. 1971 Milestones in mathematical physics: Noether's theorem. Transport Theory Statist. Phys. 1, 183-207.

TaYlor, G. I. \& Saffman, P. G. 1959 A note on the motion of bubbles in a Hele-Shaw cell and porous medium. Q. J. Mech. Appl. Maths 12, 265-279. 